MICRO- AND MACRO- SIGNATURES OF ENERGETIC CHARGED PARTICLES IN PLANETARY MAGNETOSPHERES

\author{
R.S. Selesnick \\ Space Radiation Laboratory \\ California Institute of Technology \\ Pasadena, California 91125
}

To Appear In

Advances in Space Research

SRL $92-08$

November 1992 



\title{
MICRO- AND MACRO- SIGNATURES OF ENERGETTIC CHARGED PARTICLES IN PLANETARY MAGNETOSPHERES
}

\author{
R. S. Selesnick \\ California Institute of Technology, Pasadena, CA 91125, U.S.A.
}

\begin{abstract}
Satellites orbiting within the magnetospheres of the giant planets are effective absorbers of trapped radiation, forming the characteristic and dominant structure of the radiation belts. The study of satellite absorption signatures observed by charged particle detectors involves simulating the detector response to particle distributions that include the effects of the time- and longitude-dependent absorption, guiding center drifts, and diffusion. The calculations are most easily carried out by separating solutions of the transport equation into time-dependent and -independent parts, that typically lead to small and large scale features called micro- and macro- signatures respectively. In this paper, methods for calculating the two types of signatures are described, and applied to the case of electron absorption by Saturn's satellite Mimas. The results are compared with data from Pioneer 11 and Voyager 2.
\end{abstract}

\section{INTRODUCTION}

Absorption signatures of satellites in the outer planet radiation belts have been useful tools in the study of charged particle diffusion $/ 1,2,3 /$, in discovering previously unknown orbital material $/ 4,5 /$, and in placing new constraints on planetary magnetic field models $16,7 /$. Two types of signatures have been described /8,9/. Microsignatures are small scale, transient particle depletions formed by the recent passage of a satellite through a magnetic flux shell. Macrosignatures are large scale, steady-state particle depletions formed by the average effects of satellite absorption over long time periods. Examples of the two types of signatures are shown in Figures 1 and 2, which display counting rate data from energetic particle detectors on Pioneer 11 during its encounter with Saturn. Several macrosignatures are identified in Figure 1, while the expanded scale of Figure 2 is required to easily identify a microsignature that is barely visible in Figure 1.

The next two sections describe in turn methods for simulating micro- and macro- signatures for comparison with charged particle data. They are used to construct electron absorption signatures of Saturn's satellite Mimas that are compared with the Pioneer 11 data and with data from Voyager 2. The results illustrate the first two of the three applications mentioned above.

\section{MICROSIGNATURES}

The location of a microsignature is determined by the relative positions of the satellite and spacecraft in their orbits. The condition that the hole in the electron population created by absorption drifts from the satellite to the spacecraft, over a longitude range $\delta \phi_{j}$ during a time interval $\delta t_{j}$, is

$$
\delta \phi_{j}+\omega_{d}\left(E_{j n}\right) \delta t_{j}=2 \pi n, \quad n=0,1,2,3 \cdots
$$

where the electron bounce-averaged guiding center drift frequency, $\omega_{d}$, is evaluated at the kinetic energy, $E_{j n}$, of electrons that drift $n$ times around the planet before encountering the spacecraft. The 


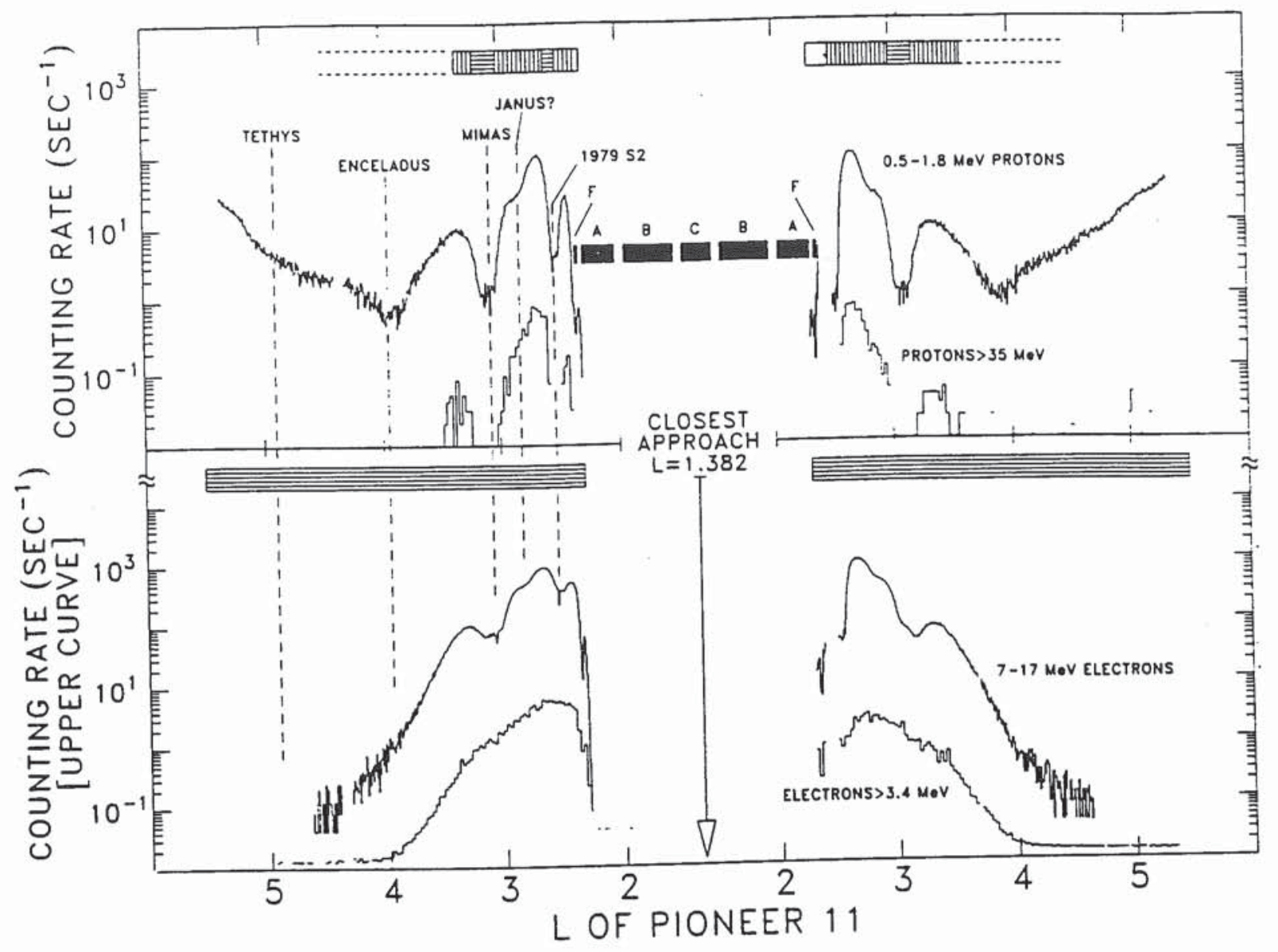

Fig. 1. Proton and electron counting rates from Pioneer 11 in Saturn's inner magnetosphere versus magnetic $L$ shell (reproduced from /4/). Inbound (left) and outbound (right) data are shown. Horizontal and vertical shading represent regions of pancake and dumbell pitchangle distributions respectively. Absorption signatures of various satellites and rings are indicated by the dashed vertical lines. The 7-17 MeV electron macrosignature of Mimas, around $L=3$ both inbound and outbound, contains an inbound microsignature that is just visible under the dashed line, but no outbound microsignature.

subscript $j$ labels the $j$ th most recent satellite encounter with the spacecraft $L$ shell prior to the spacecraft reaching that $L$ shell. Solving equation (1) for $E_{j n}$ gives the set of energies at which the electron population reaching the spacecraft will have interacted with the satellite.

At each $E_{j n}$ there is also a finite range of energies at which electrons were absorbed due to the finite size of the satellite. In the small satellite limit, this range is given by

$$
\delta E_{j n}=\frac{\Delta t_{j} \omega_{d}\left(E_{j n}\right) P_{j}}{\delta t_{j}\left|\left(\partial \omega_{d} / \partial E\right)\right|_{E_{j n}}}
$$

where $\Delta t_{j}$ is the time that the satellite spent in contact with the spacecraft $L$ shell during the $j$ th prior encounter, which is calculated from the satellite orbit. The probability of absorption on a given encounter, $P_{j}$, is unity if the particle gyroradius is negligibly small compared to the satellite radius, as 


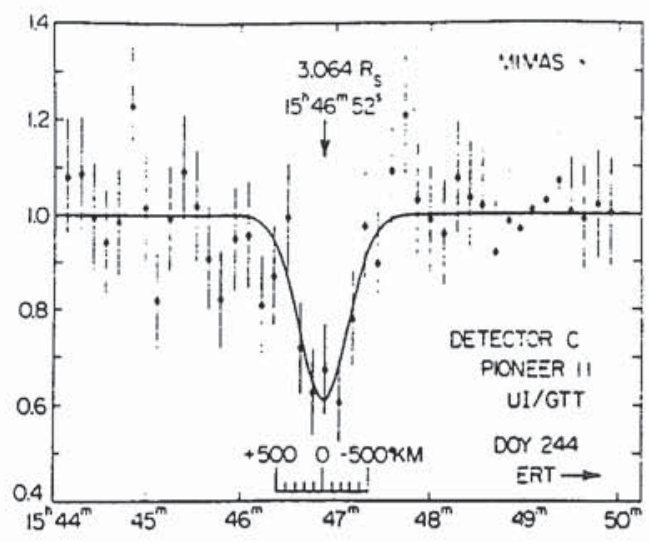

Fig. 2. Normalized counting rate profile of the microsignature observed by Pioneer 11 inbound to Saturn in the vicinity of Mimas' orbit at $L=3.07$ (reproduced from /8/).

is typical for electrons, or it can be calculated from geometrical considerations $/ 10 /$. The total $L$ shell contact time, $\Delta t$, for one complete orbit of Mimas is shown in Figure 3. The range of $L$ shells was determined by Mimas' orbital eccentricity of 0.02031, assuming that Saturn's magnetic field is that of a centered, spin-aligned dipole. Note that $\Delta t$ is sharply peaked at the minimum and maximum orbital $L$ values, so that electrons are absorbed over the largest energy ranges, and the absorption signatures can be expected to be the most prominent, at these locations.

A satellite absorbs charged particles at all energies. In the absence of diffusion, the depth of an observed microsignature is determined by its dispersion to the set of discrete energies, $E_{j n}$, due to the energy dependent electron drift as described by equations (1) and (2). Given the response function, $R(E)$, of a particle detector and the electron differential energy spectrum in the absence of satellite absorption, $j_{0}(E)$, the normalized detector counting rate for comparison with microsignature data, such as those in Figure 2, is

$$
\frac{r}{r_{0}}=1-\frac{1}{r_{0}} \sum_{j, n} R\left(E_{j n}\right) j_{0}\left(E_{j n}\right) \delta E_{j n}
$$

The normalizing rate is $r_{0}=\int R(E) j_{0}(E) d E$.

The microsignature in Figure 2 was attributed by Van Allen et al. /8/ to absorption by Mimas. From its location and shape and the lack of a microsignature of Mimas on Pioneer 11's outbound trajectory from Saturn, they concluded that the electron intensity was strongly peaked at $E_{0}=1.6 \mathrm{MeV}$. These considerations are illustrated in Figure 4, which shows the $E_{j n}$ from equation (1) and the expected microsignature from equation (3) based on an assumed differential intensity of the form

$$
j_{0}(E)=\frac{A}{E} \frac{\left(E E_{0}\right)^{\gamma}}{\left(E^{\gamma}+E_{\gamma}^{\gamma}\right)^{2}}
$$

where $A$ is a constant that does not affect the calculation. The low and high energy limits of equation (4) are $j_{0} \sim E^{\gamma-1}$ and $j_{0} \sim E^{-\gamma-1}$ respectively, so that the spectral index $\gamma$ determines the width of the intensity peak at $E=E_{0}$. In order to achieve the single, narrow inbound microsignature shown in Figure 4 a spectral index of $\gamma=80$ was required. Smaller values of $\gamma$ lead to broader and shallower microsignatures. With such a sharply peaked energy spectrum, an electron hole energy, $E_{j n}$, must 


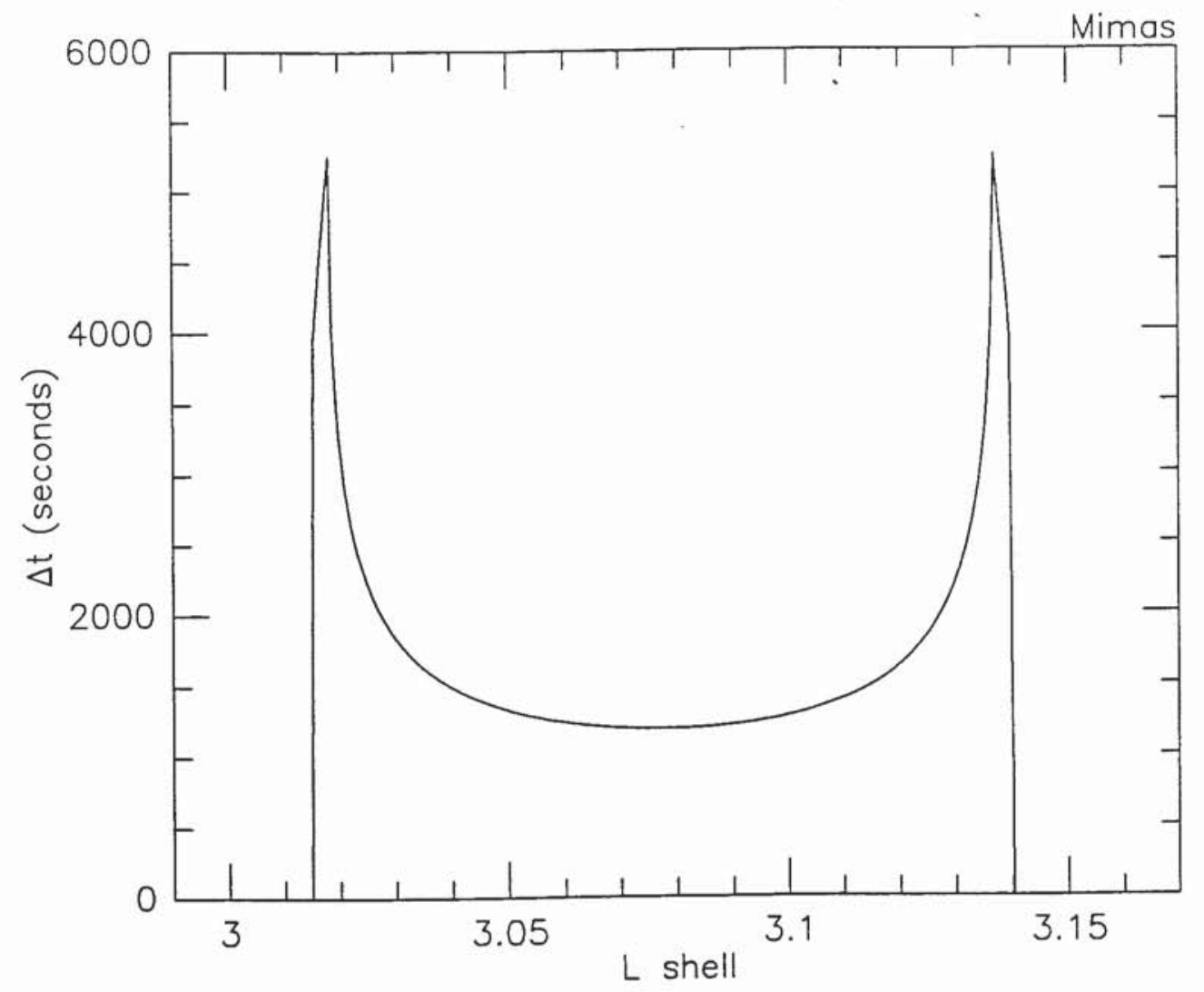

Fig. 3. $L$ shell contact time of Mimas, based on a satellite radius of $195 \mathrm{~km}$ and an orbital eccentricity of 0.02031 . The planetary magnetic field is assumed to be that of a centered, spin-aligned dipole.

occur at the energy of the spectral peak, $E_{0}$, in order to create an observable microsignature. The figure shows that this condition is true only for the inbound trajectory, so that no outbound microsignature is predicted.

The unusual, sharply peaked electron energy spectrum was attributed by Van Allen et al. to strong absorption of electrons by Enceladus, the satellite whose orbit is immediately outside that of Mimas. They assumed that only electrons whose drift period is nearly equal to the orbital period of Enceladus would not be strongly absorbed and be able to diffuse inward to Mimas. The energy of such resonant electrons upon reaching the orbit of Mimas is near the $1.6 \mathrm{MeV}$ derived from the microsignature location. If the electron energy spectrum were being so strongly influenced by Enceladus, then one may expect to see an absorption signature of that satellite. However, the data in Figure 1 show no obvious electron signature. Simpson et al. /4/ suggested that the microsignature of Figure 2 was not formed by Mimas, but by some unobserved material sharing the orbit of Mimas at an orbital phase that placed it close to Pioneer 11 on the inbound trajectory past Mimas, but not on the outbound trajectory. This interpretation of the data would not require a sharply peaked electron energy spectrum.

After Pioneer 11, Voyager 2 obtained the data shown in Figure 5. In this case microsignatures were seen on the outbound trajectory past the orbit of Mimas, but not on the inbound. Also shown in Figure 5 is an electron integral intensity spectrum compiled by Chenette and Stone /11/. The spectrum does indicate a peak in the differential intensity near the energy associated with the 


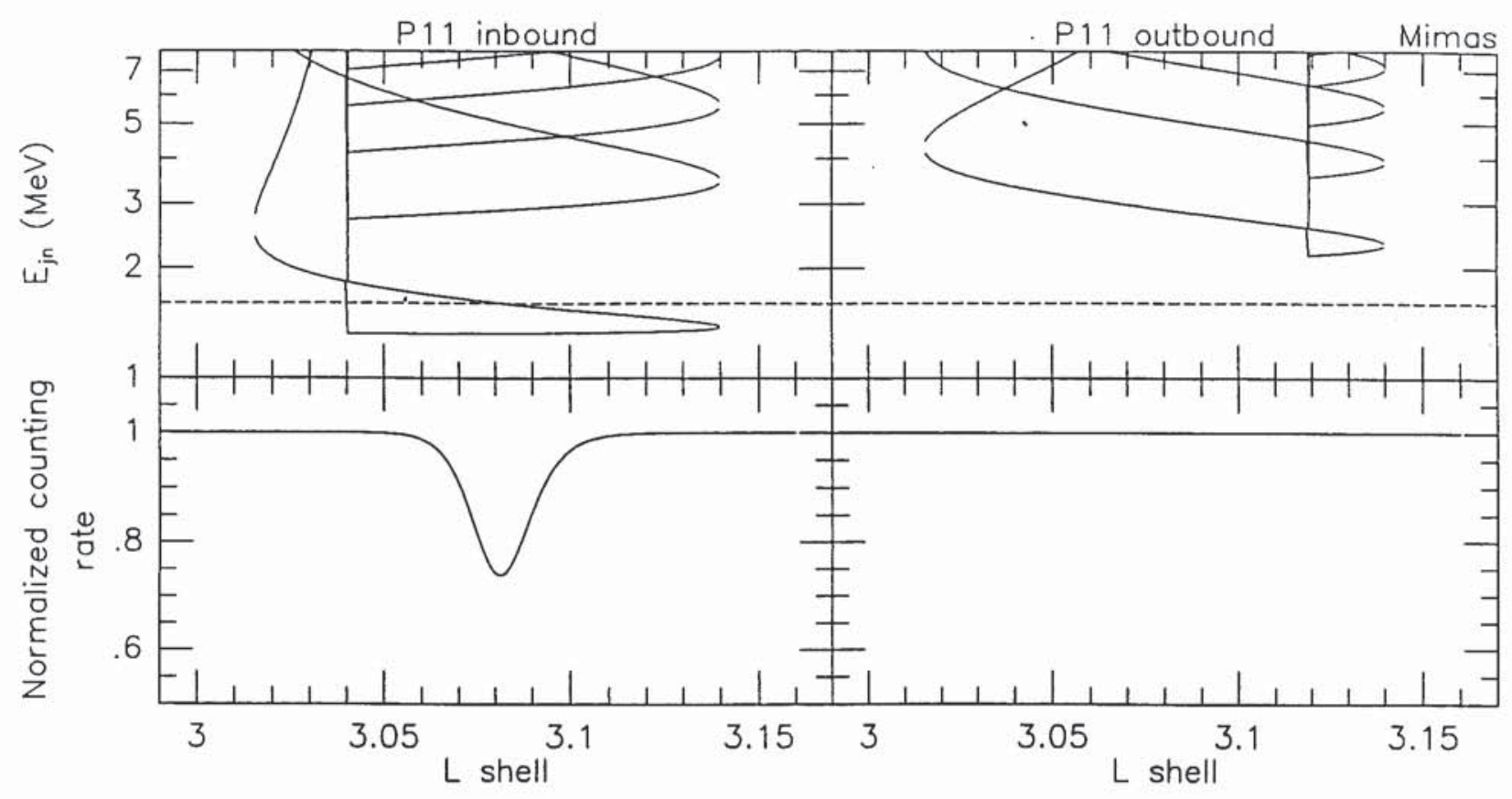

Fig. 4. (Top) Electron energies that drift from Mimas to Pioneer 11 during one orbital period of Mimas (relative to the corotating reference frame) prior to Pioneer's arrival for the inbound (left) and outbound (right) trajectories. The dashed horizontal line is at $1.6 \mathrm{MeV}$ where the electron differential energy spectrum is assumed to be sharply peaked (see text) in order to reproduce the observed inbound microsignature (Figure 2). (Bottom) Simulated normalized counting rates from equation (3). No outbound microsignature is predicted in this case.

Enceladus orbital resonance, but the spectral index, $\gamma$, is only $-5-10$. In addition, the Voyager 2 microsignatures could not have been formed by Mimas in the manner suggested by Van Allen et al. and illustrated in Figure 4, because, as in the case of the Pioneer 11 outbound trajectory, there is no $E_{j n}$ near $1.6 \mathrm{MeV}$ for either the inbound or outbound trajectories. However, with the energy spectrum shown in Figure 5, microsignatures of Mimas are predicted by equation (3). These are shown in Figure 6, where $\gamma=5$ and $E_{0}=1.6 \mathrm{MeV}$ have been adopted (a similar calculation was made by Chenette and Stone for the $n=0$ case only, but without the small satellite approximation). Since equation (3) predicts several microsignatures that were not observed, Chenette and Stone suggested that diffusion must be sufficiently fast to have filled them in. They estimated a lower limit on the radial diffusion coefficient of $D_{L L}>10^{-8} \mathrm{~s}^{-1}$.

The counting rate predictions can be modified to include the effects of diffusion:

$$
\frac{r}{r_{0}}=1-\frac{1}{r_{0}} \int d L^{\prime} \sum_{j, n} \frac{R\left(E_{j n}\right) j_{0}\left(E_{j n}\right) \delta E_{j n}}{\left(4 \pi D_{L L} \delta t_{j}\right)^{1 / 2}} e^{\frac{-\left(L-L^{\prime}\right)^{2}}{4 D_{L} \delta t_{j}}}
$$

where all $L$ dependent quantities in the integrand are evaluated at $L^{\prime}$. The Gaussian in the integrand is the response of the diffusion equation to a $\delta$-function initial condition. The time $\delta t_{j}$ since the signature was formed determines the width of the Gaussian and thereby the radial spread of the microsignature from its shape in the case of no diffusion. One-dimensional calculations of diffusional fill-in, closely related to equation (5) but not taking the drifts into account, were made by Mead and 

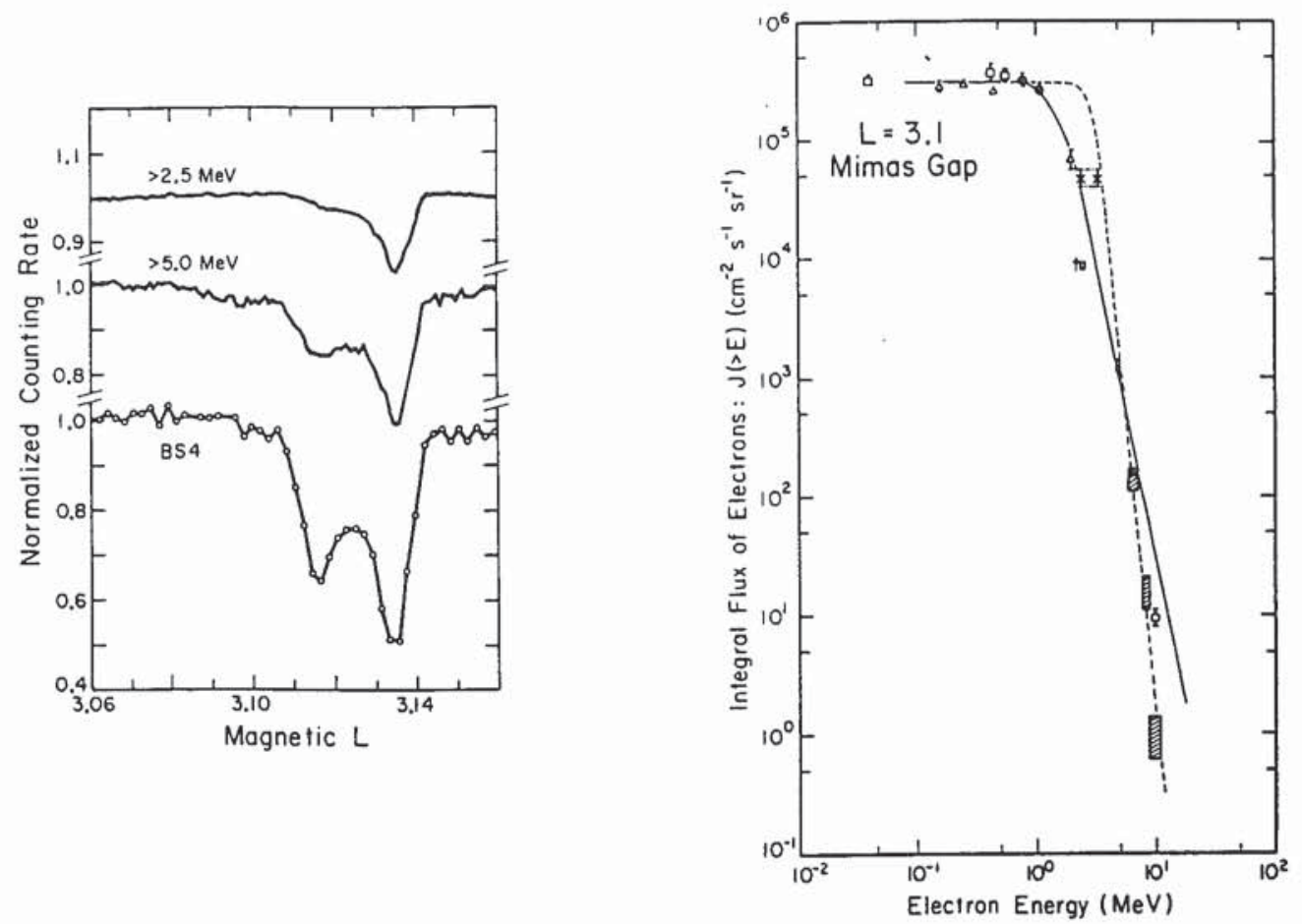

Fig. 5. (Left) Normalized counting rate profiles versus $L$ shell of the microsignatures observed by Voyager 2 outbound from Saturn in the vicinity of Mimas' orbit (reproduced from /11/). (Right) Electron integral spectrum in the vicinity of Mimas (compiled from different particle detectors and reproduced from /11/). The solid and dashed lines are model spectra based on equation (4) with $\gamma=5, E_{0}=1.6 \mathrm{MeV}$ and $\gamma=11, E_{0}=3.0 \mathrm{MeV}$, respectively.

Hess /12/ and by Van Allen et al. /8/. The generalization of their results to include drifts follows in a straightforward manner from equation (3), but the approximations involved must be addressed by considering equation (5) as a solution of a transport equation in phase space. Selesnick and Stone /9/ show that it is a reasonable approximation to neglect azimuthal diffusion, which is in the same direction as the electron drift that fills in the microsignature by energy dispersion, and also address some other approximations that are inherent in equation (5).

Counting rate predictions from equation (5) with $D_{L L}=10^{-8} \mathrm{~s}^{-1}$ are included in Figure 6. They show that the diffusion does fill in all of the Mimas microsignatures to a depth that is near the limit of a clear identification. Chenette and Stone concluded that the observed outbound microsignatures must have been the result of other orbiting material (the "Mimas ghost"), as proposed by Simpson et al. for the Pioneer 11 data. The idea is illustrated in Figure 7, which is similar to Figure 6 except that Mimas has been replaced by an hypothetical satellite sharing Mimas' orbit but at an orbital phase $170^{\circ}$ behind Mimas that caused the outbound Voyager 2 trajectory to pass by it at a longitude difference of $\delta \phi=3^{\circ}$. The inbound trajectory was separated by a further $20^{\circ}$. In addition, the satellite radius has been reduced to $25 \mathrm{~km}$ from the $195 \mathrm{~km}$ radius of Mimas. Without diffusion, deep microsignatures are predicted both inbound and outbound. With diffusion, the outbound microsignature is still deep 


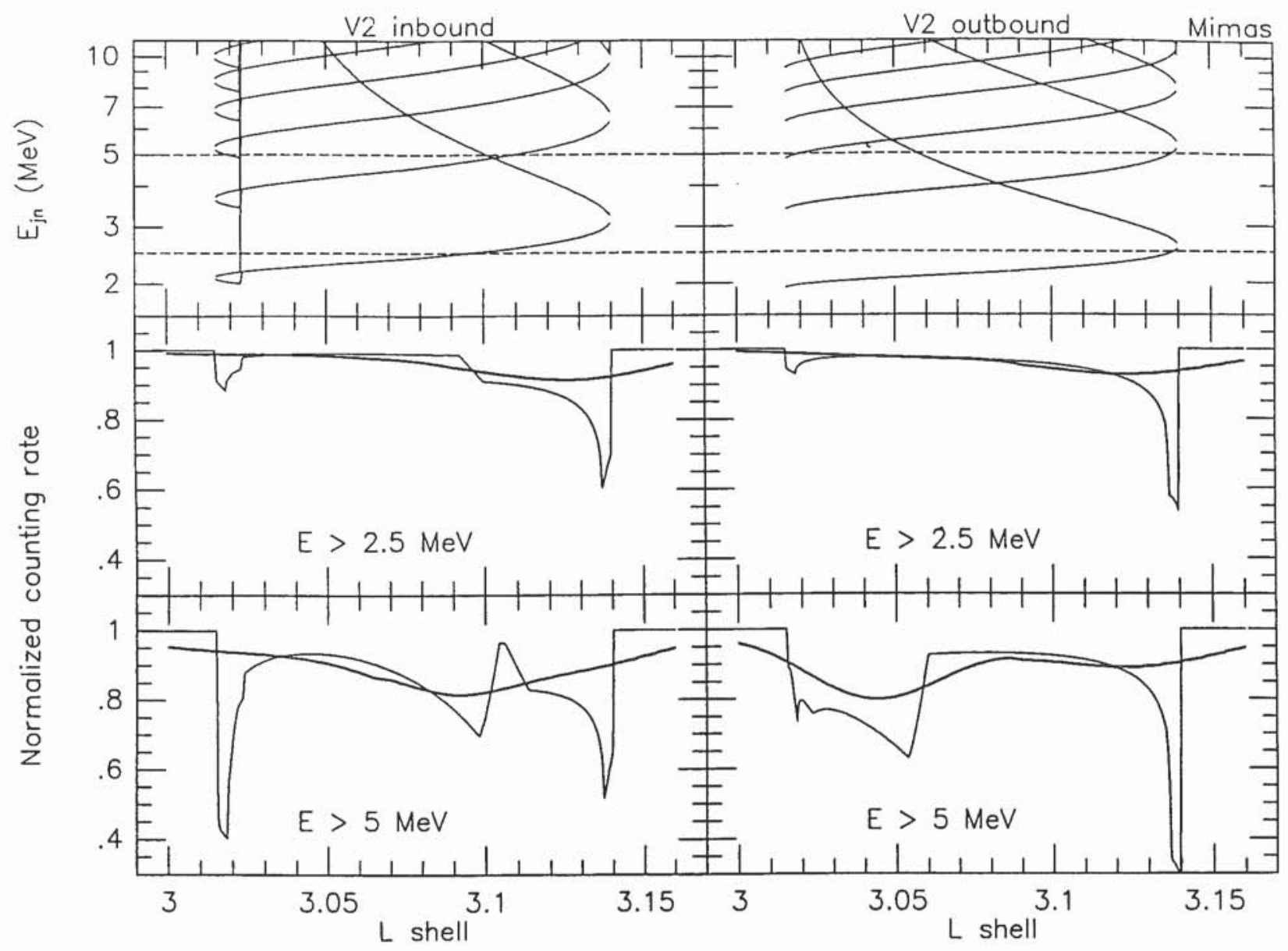

Fig. 6. Similar to Figure 4 but for the Voyager 2 trajectories past the orbit of Mimas. Normalized counting rate simulations are shown for detectors with 2.5 and $5 \mathrm{MeV}$ thresholds for comparison with the left panel of Figure 5, using the model electron spectrum shown by the solid line in the right panel of Figure 5. The energy thresholds are indicated by the dashed lines in the top panels. Simulations are shown for the case without diffusion (thin lines) based on equation (3), and with diffusion (thick lines) based on equation (5) and $D_{L L}=10^{-8} \mathrm{~s}^{-1}$.

due to the recent passage of the satellite, but the inbound microsignature has been substantially filled in during the longer time since it was formed. The width of the outbound microsignature is narrower than observed, even with diffusion, and Chenette and Stone proposed that it may have been formed by an extended cloud of small satellites. Although the ghost hypothesis can apparently explain the Voyager 2 data, a separate ghost would probably be required to explain the Pioneer 11 microsignature if that were not due to Mimas, because it was observed at a substantially different longitude relative to Mimas.

\section{MACROSIGNATURES}

Over a period of many satellite orbits the steady-state macrosignature is formed as a balance between the cumulative particle losses due to satellite absorption and their replenishment by radial diffusion. The substantially larger scale of the macrosignature allows it to remain stable while the microsignatures are rapidly filled in by diffusion. However, the clear separation of the total absorption signature into the micro- and macro- components is an approximation that may not be valid in every situation /9/. 


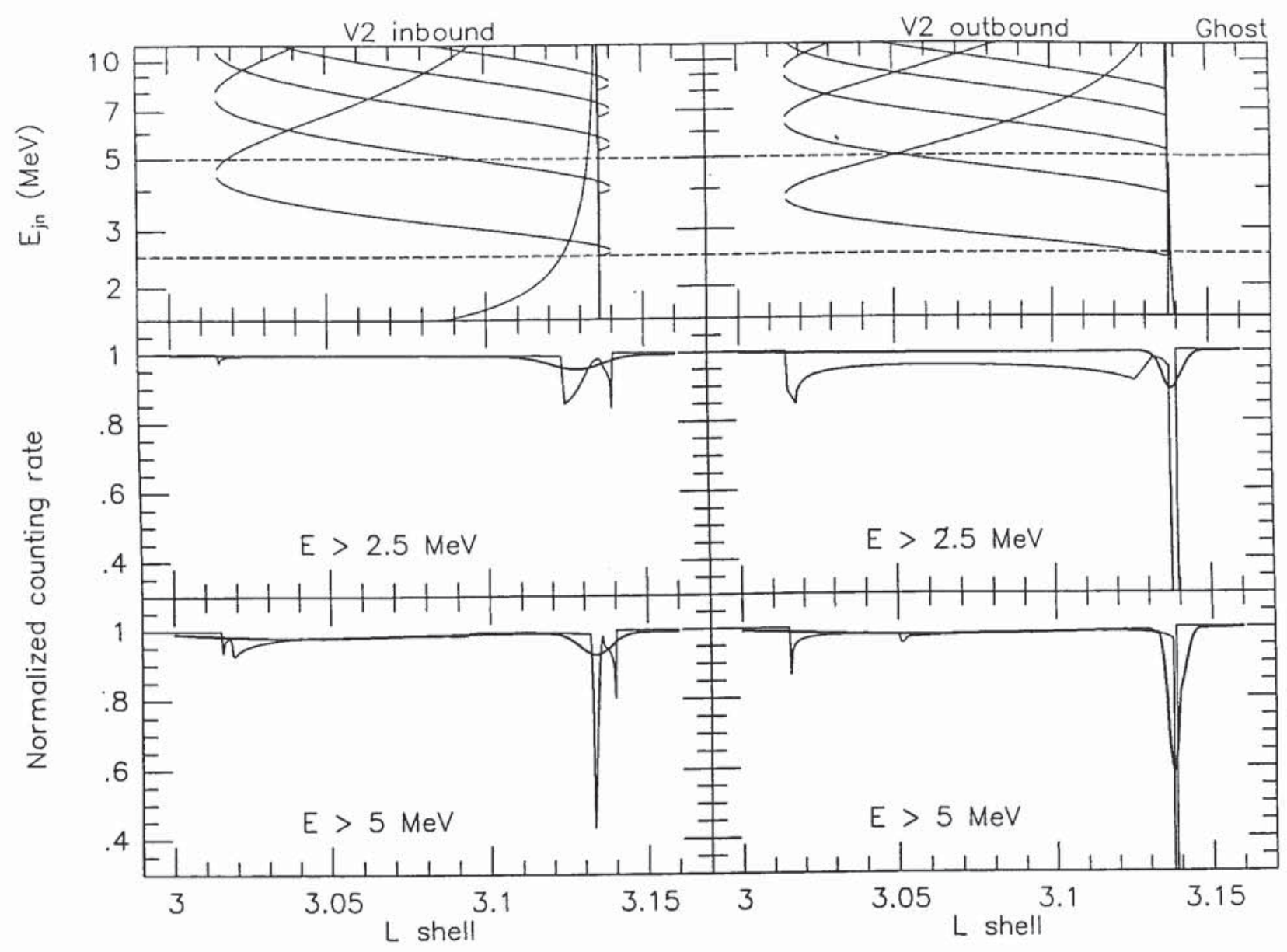

Fig. 7. Similar to Figure 6, but the calculations are based on the orbit of Mimas shifted in phase by $170^{\circ}$ to simulate the microsignatures of an hypothetical "Mimas ghost" satellite with a radius of $25 \mathrm{~km}$.

The satellite absorption loss rate, or inverse lifetime, averaged over time and longitude, is /9/

$$
\left\langle\tau_{S}^{-1}\right\rangle=\sum_{j} \frac{\Delta t_{j} \omega_{d}}{2 \pi T} P_{j}
$$

where the summation extends over all $L$ shell encounters during the satellite orbital period, $T$, in the corotating reference frame. The loss rate determines the phase-space density, $f=j / p^{2}$ where $p$ is momentum, which is obtained as a solution of the steady-state radial diffusion equation,

$$
L^{2} \frac{\partial}{\partial L}\left[\frac{D_{L L}}{L^{2}} \frac{\partial f}{\partial L}\right]=\left\langle\tau_{S}^{-1}\right\rangle f
$$

Assuming that the diffusion is caused by violation of the third adiabatic invariant, equation (7) applies to $f$ evaluated at constant values of the first two adiabatic invariants. Typically equation (7) is solved numerically as a boundary value problem, with an outer boundary condition taken from the data and an inner boundary condition of $f=0$ near the planet. The macrosignature is constructed by converting to a simulated counting rate, $r=\int R(E) j(E) d E$ with $j=p^{2} f$. Note that the intensity $j$ and the response function $R$ may be functions of pitch-angle as well as energy, in which case the counting rate integral must also extend over pitch angle. The averaging procedure for deriving equation (7) from the time and longitude dependent transport equation is discussed by Selesnick and Stone /9/. 


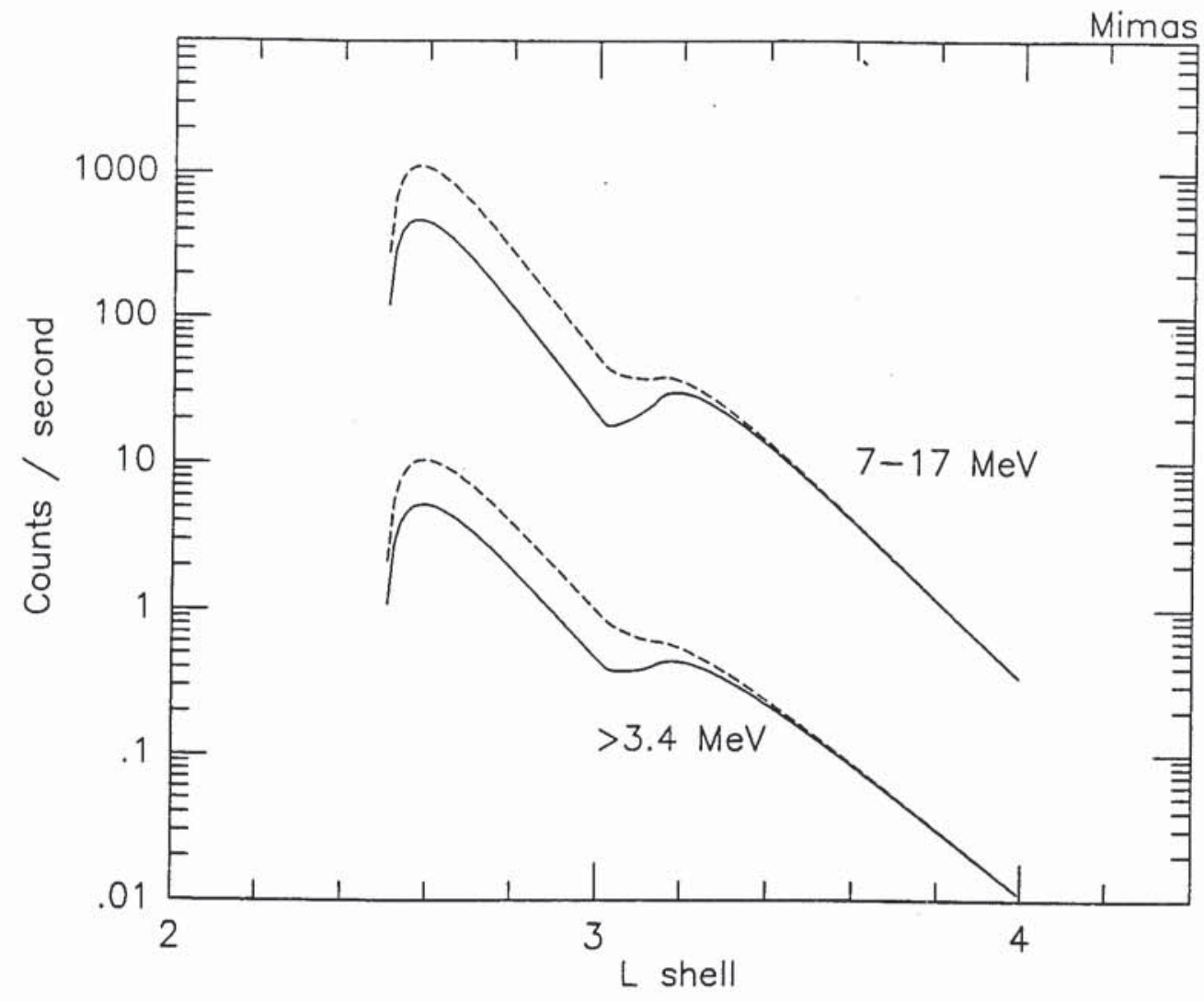

Fig. 8. Simulated electron counting rates based on solutions of the diffusion equation (7), for comparison with the Mimas macrosignatures in Figure 1. The solid curves are based on a radial diffusion coefficient of $D_{L L}=10^{-8}(L / 3)^{7} \mathrm{~s}^{-1}$ and the dashed curves on $D_{L L}=2 \times 10^{-8}(L / 3)^{7} \mathrm{~s}^{-1}$.

Simulations of the Mimas macrosignature, for comparison with the electron counting rate data of Figure 1, are shown in Figure 8. The simulations assume that Pioneer 11 was in the magnetic equatorial plane, which is approximately correct, that $j=A E^{-\gamma} \sin ^{2} \alpha$, where $\alpha$ is the electron pitchangle, and that the detector response functions are omnidirectional. The spectral indices are constrained by the radial gradients of the counting rates, and values of $\gamma=16$ for the $7-17 \mathrm{MeV}$ rate and $\gamma=12$ for the $>3.4 \mathrm{MeV}$ rate were adopted. The simulations based on $D_{L L}=10^{-8}(L / 3)^{7} \mathrm{~s}^{-1}$ show that the observed depth and extent of the 7-17 MeV macrosignature are approximately reproduced, but that the $>3.4 \mathrm{MeV}$ simulation predicts a macrosignature that was not seen in the data. The simulations with $D_{L L}=2 \times 10^{-8}(L / 3)^{7} \mathrm{~s}^{-1}$ show that the $>3.4 \mathrm{MeV}$ macrosignature is substantially filled in with the higher diffusion coefficient, but that the $7-17 \mathrm{MeV}$ macrosignature is then not deep enough. Therefore the simulations suggest that the diffusion coefficient decreases with increasing electron energy, as may be expected for many diffusion mechanisms, such as that due to atmospheric dynamo electric fields $/ 2 /$. Also, these simulations lead to diffusion coefficients that are approximately consistent with the lower limit of $D_{L L}>10^{-8} \mathrm{~s}^{-1}$ derived from the microsignature data. Note that the simulations include losses due to Mimas only. If there are significant losses from other material in the Mimas orbit then the diffusion coefficients would have to be correspondingly higher to reproduce the observed macrosignatures. 


\section{SUMMARY AND CONCLUSION}

Simple methods have been described for simulating satellite micro- and macro- signatures as observed by charged particle detectors, including the effects of particle drifts and radial diffusion. They have been applied to the interpretation of absorption signatures that were observed in the vicinity of Saturn's satellite Mimas. The results confirm that the observed microsignatures are likely to have been produced by orbital material other than Mimas, and that both micro- and macro- signatures are consistent with a radial diffusion coefficient near $10^{-8} \mathrm{~s}^{-1}$. Many other examples of absorption signatures in the radiation belts of the outer planets, both of satellites and rings, are available in the literature. They provide a valuable tool in magnetospheric and planetary physics. Future improvements in their theoretical interpretation may address such issues as the importance of particle diffusion in pitch-angle and energy.

\section{ACKNOWLEDGEMENTS}

The idea of further investigating the interpretation of the Mimas microsignatures was suggested to me by Chris Goertz during fruitful discussions that we had on this subject. This work was supported by NASA under contract NAS7-918 and grant NAGW-2402.

\section{REFERENCES}

1. M. F. Thomsen, Rev. Geophys. 17, 369 (1979).

2. L. L. Hood, J. Geophys. Res. 94, 15,077 (1989).

3. R. S. Selesnick, E. C. Stone, J. Geophys. Res. 96, 5651 (1991).

4. J. A. Simpson, T. S. Bastian, D. L. Chenette, R. B. McKibben, K. R. Pyle, J. Geophys. Res. 85, 5731 (1980).

5. J. N. Cuzzi, J. A. Burns, Icarus 74, 284 (1988).

6. D. L. Chenette, L. Davis, Jr., J. Geophys. Res. 87, 5267 (1982).

7. R. S. Selesnick, J. Geophys. Res., 97, 10,857 (1992).

8. J. A. Van Allen, M. F. Thomsen, B. A. Randall, J. Geophys. Res. 85, 5709 (1980).

9. R. S. Selesnick, E. C. Stone, J. Geophys. Res. 96, 19,137 (1991).

10. M. T. Paonessa, A. F. Cheng, J. Geophys. Res. 90, 3428 (1985).

11. D. L. Chenette, E. C. Stone, J. Geophys. Res. 88, 8755 (1983).

12. G. P. Mead, W. N. Hess, J. Geophys. Res. 78, 2793 (1973). 